\title{
An exactly solvable model for depletion phenomena
}

\author{
H.N.W. Lekkerkerker*, B. Widom ${ }^{1}$ \\ Van't Hoff Laboratorium, Debye Research Institute, Utrecht University, Padualaan 8 , \\ 3584 CH Utrecht, The Netherlands
}

Received 14 March 2000; accepted 26 April 2000

\begin{abstract}
The equilibrium properties of a one-dimensional mixture of hard rods of two different lengths are obtained. These are used to test the free-volume approximation, which has in the past been applied in the study of hard-sphere mixtures in three dimensions. Three different versions of the free-volume approximation are tested. In the most primitive of these the smaller spheres (or rods) are assumed not to interact with each other. In higher levels of the approximation the interactions between the smaller molecules are partly taken into account. The results show the ranges of compositions and densities over which the approximations are accurate and they identify one form of the approximation as the most reliable. (c) 2000 Elsevier Science B.V. All rights reserved.
\end{abstract}

PACS: 05.20.Jj; 05.70.Ce; 05.70.Fh; 05.70.Jk

Keywords: Depletion interaction; Hard-sphere mixtures; Free-volume approximation

\section{Introduction}

In the last 25 years, a large interest has developed for phase separation processes induced by entropic interactions. In 1954, Asakura and Oosawa [1] showed that when two large (colloidal) bodies are immersed in a solvent with non-adsorbing polymers, an effective, attractive interaction is induced between the two bodies due to an unbalanced osmotic pressure arising from the depletion of the polymers in the region between the two bodies. This result was independently recovered and further elaborated by Vrij [2]

\footnotetext{
* Corresponding author.

E-mail address: h.n.w.lekkerkerker@chem.uu.nl (H.N.W. Lekkerkerker).

${ }^{1}$ Permanent address: Department of Chemistry, Baker Laboratory, Cornell University, Ithaca, NY 14853, USA.
} 
in 1976. The attractive interaction, which is called depletion interaction, is the origin of the rich phase behaviour displayed by colloid-polymer mixtures.

In the 1980s, Gast et al. [3] employed an effective-pairwise-potential model based on the Asakura-Oosawa depletion potential to calculate the phase equilibria for colloidpolymer mixtures. Later, Lekkerkerker et al. [4] proposed a free-volume approach to calculate the phase behaviour of colloid-polymer mixtures. The key quantity in this approach is the statistically averaged volume that is available for the polymers at a given polymer activity set by a hypothetical reservoir. In this approach the partitioning of polymer between the colloid-rich and colloid-poor phases is taken into account. The calculated phase diagrams have been confirmed by experiments $[5,6]$. The free-volume theory for colloid-polymer phase behaviour has been compared with simulation $[7,8]$ as well.

The free-volume approach can also be applied to the phase behaviour of highly asymmetric binary hard-sphere mixtures [9,10], for which a demixing transition was first conjectured by Biben and Hansen [11]. These predictions have stimulated experimental work [12-14], theoretical analyses [15], and simulations [15-18]. Here we analyse a solvable one-dimensional model of a binary mixture of hard rods. Of course there are no phase transitions in this model, but a comparison between the free-volume model and exact analytical results can be made. This allows one to judge the ranges of compositions and densities over which the free-volume approximation may be a reliable guide to the properties of hard-sphere mixtures.

\section{Free-volume approximations}

The larger spheres are here identified by subscript 1 and the smaller ones by subscript 2. The basic form of the approximation in three dimensions is to take the configuration-space integral in the volume $V$ to be the product of two factors: (i) the configuration integral for the $N_{1}$ larger spheres alone, as though they were unperturbed by the presence of the smaller; and (ii) that for the $N_{2}$ smaller spheres as though they did not interact with each other and their centers had free access to the mean free volume, $v\left(N_{1}, V\right)$, in the typical equilibrium configuration of the unperturbed larger spheres. Thus, the approximation for the mixture's canonical partition function $Q\left(N_{1}, N_{2}, V, T\right)$ at the temperature $T$ in terms of the partition function $Q^{0}\left(N_{1}, V, T\right)$ for the larger spheres alone in the volume $V$, is

$$
Q\left(N_{1}, N_{2}, V, T\right) \simeq Q^{0}\left(N_{1}, V, T\right) \frac{v\left(N_{1}, V\right)^{N_{2}}}{\Lambda_{2}^{3 N_{2}} N_{2} !}
$$

where $\Lambda_{2}$ is the conventional thermal de Broglie wavelength of the type- 2 molecules. The Helmholtz free energy $F\left(N_{1}, N_{2}, V, T\right)$ of the mixture is then related to that for the $N_{1}$ larger spheres alone in the volume, $F^{0}\left(N_{1}, V, T\right)$, by

$$
F\left(N_{1}, N_{2}, V, T\right) \simeq F^{0}\left(N_{1}, V, T\right)-N_{2} \mathrm{k} T \ln \frac{\mathrm{e} v}{\Lambda_{2}^{3} N_{2}} .
$$


Note that $v$ is itself $V$ times a function only of the density $\rho_{1}=N_{1} / V$ of the larger spheres. The chemical potential $\mu_{2}$ of the smaller spheres, and the corresponding activity $z_{2}$ defined so as to become asymptotically equal to the number density $\rho_{2}=N_{2} / V$ in the dilute-gas limit, are then, in this approximation,

$$
\mu_{2}=\mathrm{k} T \ln \left(\Lambda_{2}^{3} z_{2}\right), \quad z_{2} \simeq \frac{N_{2}}{v},
$$

while the chemical potential $\mu_{1}\left(\rho_{1}, \rho_{2}, T\right)$ of the larger spheres in the mixture is related to that of those spheres alone in the volume, $\mu_{1}^{0}\left(\rho_{1}, T\right)$, by

$$
\mu_{1}\left(\rho_{1}, \rho_{2}, T\right) \simeq \mu_{1}^{0}\left(\rho_{1}, T\right)-\frac{N_{2}}{v} \mathrm{k} T \frac{\mathrm{d} v / V}{\mathrm{~d} \rho_{1}} .
$$

Since $v$ has been defined as the free volume experienced by hypothetically noninteracting spheres of species 2 in the hypothetically unperturbed system of the $N_{1}$ larger spheres in the volume $V$, it is the same as the free volume that would be measured by a single smaller sphere acting as a test particle in the fluid of the $N_{1}$ larger spheres. The quantity $v / V$ in (4) is thus the Henry's law constant of the small spheres as a dilute solute in the fluid of large spheres as solvent. We retain the symbol $v$ for that free volume in all that follows. The quantity $N_{2} / v$ in (3) is then the Henry's law (dilute-solution) approximation to the activity $z_{2}$.

One may now imagine that the approximation in (4) would be improved if the coefficient $N_{2} / v$ of the second term on the right-hand side were identified with the exact activity of species 2 in the mixture, for in that way the effect of the interactions among the smaller spheres would be partially taken into account. One may imagine a reservoir of the type- 2 spheres in osmotic equilibrium with the mixture; the exact activity of the small spheres in the mixture is accordingly denoted $z_{2}^{\mathrm{R}}$ here. Thus, as a putative improvement of approximation (4), one could take

$$
\mu_{1} \simeq \mu_{1}^{0}\left(\rho_{1}, T\right)-z_{2}^{\mathrm{R}} \mathrm{k} T \frac{\mathrm{d} v / V}{\mathrm{~d} \rho_{1}} .
$$

Like the earlier (4), this, too, may be viewed as a perturbation approximation, in which, to a first approximation, the larger spheres are taken to be unperturbed by the presence of the smaller, while the second term on the right-hand side is then a correction. In (4) the correction is linear in $N_{2}$ while (5) is an attempt to incorporate some higher-order effects.

While the smaller spheres were still being taken to be non-interacting, i.e., before $N_{2} / v$ in (4) was replaced by $z_{2}^{\mathrm{R}}$ in (5), the pressure $p^{\mathrm{R}}$ in the reservoir would have been related to $z_{2}$ by the ideal gas $p^{\mathrm{R}}=z_{2} \mathrm{k} T$. Thus, still another way of taking account of the interactions among the small spheres would be to replace (4) by

$$
\mu_{1} \simeq \mu_{1}^{0}\left(\rho_{1}, T\right)-p^{\mathrm{R}} \frac{\mathrm{d} v / V}{\mathrm{~d} \rho_{1}},
$$

where now $p^{\mathrm{R}}$ is the pressure of the pure fluid of species 2 at the exact activity $z_{2}^{\mathrm{R}}$ of that component in the mixture.

The approximations (4), (5), and (6) are three alternative, inequivalent versions of the free-volume approximation, and will be denoted II, III, and IV in what follows, 
while I will refer to the true properties of the mixture. These are the approximations that are tested in the exactly solvable one-dimensional model.

\section{One-dimensional model}

We have $N_{1}$ hard rods of length $a$ and $N_{2}$ of length $b$ on a line of length $L>N_{1} a+$ $N_{2} b$. The configuration-space integral proves to be independent of the ordering of the rods on the line, with the result that the partition function $Q\left(N_{1}, N_{2}, L, T\right)$ is just

$$
Q\left(N_{1}, N_{2}, L, T\right)=\frac{1}{\Lambda_{1}^{N_{1}} \Lambda_{2}^{N_{2}} N_{1} ! N_{2} !}\left[L-\left(N_{1} a+N_{2} b\right)\right]^{N_{1}+N_{2}},
$$

with $\Lambda_{1}$ and $\Lambda_{2}$ the respective de Broglie wavelengths of the two kinds of particles. With the linear densities $\rho_{1}=N_{1} / L$ and $\rho_{2}=N_{2} / L$, the Helmholtz free energy $F\left(N_{1}, N_{2}, L, T\right)$ at temperature $T$ is then

$$
\begin{aligned}
F\left(N_{1}, N_{2}, L, T\right)= & -\left(N_{1}+N_{2}\right) \mathrm{k} T \ln \left\{\mathrm{e}\left[1-\left(a \rho_{1}+b \rho_{2}\right)\right]\right\} \\
& +\mathrm{k} T\left[N_{1} \ln \left(\Lambda_{1} \rho_{1}\right)+N_{2} \ln \left(\Lambda_{2} \rho_{2}\right)\right]
\end{aligned}
$$

and the chemical potentials $\mu_{1}\left(\rho_{1}, \rho_{2}, T\right)$ and $\mu_{2}\left(\rho_{1}, \rho_{2}, T\right)$ are given by

$$
\begin{aligned}
& \frac{\mu_{1}}{\mathrm{k} T}=\ln \frac{\Lambda_{1} \rho_{1}}{1-\left(a \rho_{1}+b \rho_{2}\right)}+\frac{a\left(\rho_{1}+\rho_{2}\right)}{1-\left(a \rho_{1}+b \rho_{2}\right)}, \\
& \frac{\mu_{2}}{\mathrm{k} T}=\ln \frac{\Lambda_{2} \rho_{2}}{1-\left(a \rho_{1}+b \rho_{2}\right)}+\frac{b\left(\rho_{1}+\rho_{2}\right)}{1-\left(a \rho_{1}+b \rho_{2}\right)} .
\end{aligned}
$$

We introduce the "volume" fractions

$$
\eta_{1}=a \rho_{1}, \quad \eta_{2}=b \rho_{2}
$$

and the size ratio

$$
q=b / a \text {. }
$$

Then from (9) and (10) the activities $z_{1}\left(\eta_{1}, \eta_{2}\right)=\Lambda_{1}^{-1} \exp \left(\mu_{1} / \mathrm{k} T\right)$ and $z_{2}\left(\eta_{1}, \eta_{2}\right)=$ $\Lambda_{2}^{-1} \exp \left(\mu_{2} / \mathrm{k} T\right)$, which become asymptotically equal to $\rho_{1}$ and $\rho_{2}$, respectively, in the dilute-gas limit, are given by

$$
a z_{1}=\frac{\eta_{1}}{1-\left(\eta_{1}+\eta_{2}\right)} \mathrm{e}^{\left(\eta_{1}+\eta_{2} / q\right) /\left(1-\left(\eta_{1}+\eta_{2}\right)\right)}, \quad b z_{2}=\frac{\eta_{2}}{1-\left(\eta_{1}+\eta_{2}\right)} \mathrm{e}^{\left(q \eta_{1}+\eta_{2}\right) /\left(1-\left(\eta_{1}+\eta_{2}\right)\right)} .
$$

The pressure $p\left(\rho_{1}, \rho_{2}, T\right)$, found either from (8) as $p=-(\partial F / \partial L)_{N_{1}, N_{2}, T}$ or from (8), (9), and (10) as $p=\left(N_{1} \mu_{1}+N_{2} \mu_{2}-F\right) / L$, is

$$
p=\frac{\rho_{1}+\rho_{2}}{1-\left(\eta_{1}+\eta_{2}\right)} \mathrm{k} T,
$$

as obtained also by Lebowitz et al. [19]. It is a simple generalization of the well-known $p=\rho_{1} \mathrm{k} T /\left(1-a \rho_{1}\right)$ for the one-component case $\left(\rho_{2}=0\right)$. 
We take $q<1$, thus identifying the smaller rods as the species 2 . The free volume $v$ was defined in Section 2 in such a way as to be identified with the ratio $N_{2} / z_{2}$ in the limit in which the component 2 is infinitely dilute in 1 . Then here, from (13),

$$
\frac{v}{L}=\left(1-\eta_{1}\right) \mathrm{e}^{-q \eta_{1} /\left(1-\eta_{1}\right)} \text {. }
$$

The quantity called $z_{2}^{\mathrm{R}}$ in Section 2 is the exact activity of component 2 in the mixture and is thus the $z_{2}$ given by the second of Eqs. (13). The quantity called $p^{\mathrm{R}}$ in Section 2 is the pressure of hypothetically pure 2 in the "reservoir" at activity $z_{2}^{\mathrm{R}}$ and temperature $T$. From (13) and (14), and in accord with the known result $z=(p / \mathrm{k} T) \exp (b p / \mathrm{k} T)$ for pure hard rods of length $b$,

$$
b z_{2}^{\mathrm{R}}=\frac{b p^{\mathrm{R}}}{\mathrm{k} T} \mathrm{e}^{b p^{\mathrm{R}} / \mathrm{k} T}=\frac{1}{1 / y-1} \mathrm{e}^{(x+y) /(1-y)},
$$

where we have introduced the abbreviations,

$$
x=\frac{q \eta_{1}}{1-\eta_{1}}, \quad y=\frac{\eta_{2}}{1-\eta_{1}} .
$$

We note that $\eta_{1}+\eta_{2} \leqslant 1$ so $y \leqslant 1$. With the function $W(x)$ (Lambert's $W$ function [20]) defined by $W(u) \exp [W(u)] \equiv u$, we may express $b p^{\mathrm{R}} / \mathrm{k} T$ from (16) as

$$
\frac{b p^{\mathrm{R}}}{\mathrm{k} T}=W\left(\frac{1}{1 / y-1} \mathrm{e}^{(x+y) /(1-y)}\right) .
$$

From Eq. (9) for $\mu_{1}$ and the abbreviations in (12) and (17), the exact $\left(\mu_{1}-\mu_{1}^{0}\right) / \mathrm{k} T$, to be denoted $\mathrm{I}$, is given by

$$
\frac{\mu_{1}-\mu_{1}^{0}}{\mathrm{k} T}=-\ln (1-y)+\frac{1}{q} \frac{x+1}{1 / y-1} \quad \text { (I), }
$$

while from (15), (16), and (18), approximations (4), (5), and (6) (now with $V=L$ ), denoted II, III, and IV, respectively, are given by

$$
\begin{aligned}
& \frac{\mu_{1}-\mu_{1}^{0}}{\mathrm{k} T} \simeq\left(1+\frac{x+1}{q}\right) y \quad(\mathrm{II}) \\
& \frac{\mu_{1}-\mu_{1}^{0}}{\mathrm{k} T} \simeq\left(1+\frac{x+1}{q}\right) \frac{1}{1 / y-1} \mathrm{e}^{(x+1) /(1 / y-1)} \quad(\mathrm{III}), \\
& \frac{\mu_{1}-\mu_{1}^{0}}{\mathrm{k} T} \simeq\left(1+\frac{x+1}{q}\right) \mathrm{e}^{-x} W\left(\frac{1}{1 / y-1} \mathrm{e}^{(x+y) /(1-y)}\right)
\end{aligned}
$$

in this one-dimensional mixture model. The object is to compare approximations II-IV with the exact I. This is done in the next section.

The exact (19) and approximations (20)-(22) all express $\left(\mu_{1}-\mu_{1}^{0}\right) / \mathrm{k} T$ as a function of the composition $\rho_{1}, \rho_{2}$ (or equivalently $\eta_{1}, \eta_{2}$ or $x, y$ ) of the mixture. In applications of these mean-field, free-volume approximations to mixtures of spheres in three dimensions it is important for studying the phase behaviour also to know $\left(\mu_{1}-\mu_{1}^{0}\right) / \mathrm{k} T$ as a function of $\rho_{1}$ and $z_{2}$, i.e., of $\rho_{1}$ and $z_{2}^{\mathrm{R}}$ or $p^{\mathrm{R}} / \mathrm{k} T$. To test this version of the 


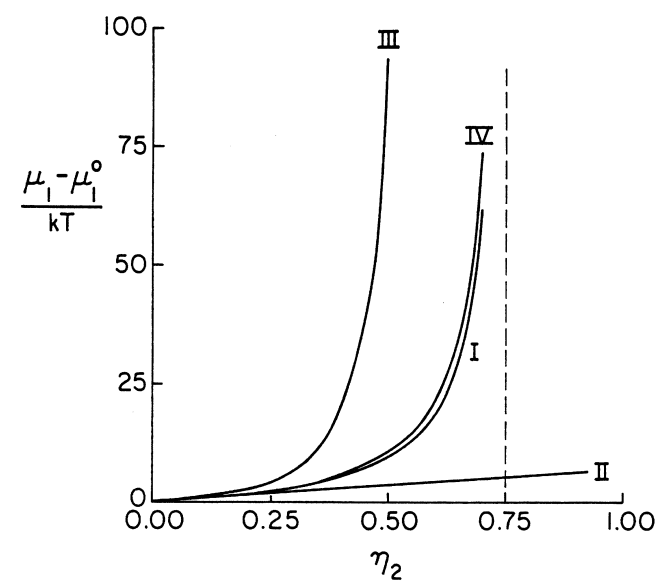

Fig. 1. $\left(\mu_{1}-\mu_{1}^{0}\right) / \mathrm{k} T$ as a function of the volume fraction $\eta_{2}$ for fixed volume fraction $\eta_{1}=\frac{1}{4}$ and size ratio $q=b / a=\frac{1}{4}$. The curve marked I is the exact result for the hard-rod model; II, III, and IV are the approximations in Eqs. (4), (5), and (6) [Eqs. (20), (21), (22) in the one-dimensional model], respectively.

approximation the $y$ that appears in (19)-(22) is to be understood as that function of $x$ and $b z_{2}^{\mathrm{R}}$ or $b p^{\mathrm{R}} / \mathrm{k} T$ that is given implicitly by (16). This test, too, is made in the next section.

\section{Test of the approximations}

In Fig. 1 are shown $\left(\mu_{1}-\mu_{1}^{0}\right) / \mathrm{k} T$, from (19)-(22), as functions of $\eta_{2}$ with the fixed values $q=\frac{1}{4}$ and $\eta_{1}=\frac{1}{4}$. The curve marked $\mathrm{I}$ is the exact result for this one-dimensional system, from (19), while II, III, and IV are the approximations as given by (20), (21), and (22), respectively. With the fixed $\eta_{1}=\frac{1}{4}$, the value $\eta_{2}=\frac{3}{4}$ corresponds to the close packing of the rods on the line. The ordinates of the curves I, III, and IV are all infinite at that point; the vertical dashed line in the figure is the asymptote. The line II comes from the approximation in which no account is taken of the interactions of the particles of species 2 with each other, so it does not recognize the condition of close packing and can extend beyond $\eta_{2}=\frac{3}{4}$.

The approximations are better the smaller $q$ is and they become exact when $q=0$, i.e., when the smaller particles are points. The fixed value $q=\frac{1}{4}$ in Fig. 1 is greater than the value it has in most applications, while the fixed value $\eta_{1}=\frac{1}{4}$ is in the mid-range of practical values of $\eta_{1}$. Thus, Fig. 1 may be taken as a fair test of the reliability of the various approximations.

All four of I-IV are the same to first order in $\eta_{2}$ but II and III rapidly depart from the exact I as $\eta_{2}$ increases. The most rapid departure is that of III, which grows exponentially rapidly. That is the approximation in which one attempts to account for the interactions of the smaller particles with each other by replacing $N_{2} / v$ in (4) by the exact activity $z_{2}^{\mathrm{R}}$ of component 2 in the mixture. As the volume fraction $\eta_{2}$ increases 
it becomes rapidly more difficult for a test particle to find a hole in the "reservoir" of type- 2 particles, which is the reason for the exponential growth of $z_{2}^{\mathrm{R}}$ with $\eta_{2}$. This exponential growth is seen explicitly for the one-dimensional model in (13).

The pressure $p^{\mathrm{R}}$, by contrast, grows much more slowly with increasing $\eta_{2}$ : only as the logarithm of $z_{2}^{\mathrm{R}}$, as seen explicitly for the one-dimensional model in (16). This results in the approximation IV remaining very accurate until very close to the close-packed limit, as seen in the figure. At $\eta_{2}=\frac{1}{2}$, so at a total volume fraction $\eta_{1}+\eta_{2}$ that is $\frac{3}{4}$ of that of close packing, the exact I and approximate IV differ by only $9 \%$; and even at $\eta_{2}=0.70$, so at a total volume fraction that is $95 \%$ of that of close packing, they differ by only $16 \%$.

As remarked in Section 3, one wishes also to test the free-volume approximation for $\mu_{1} / \mathrm{k} T$ as a function of $\rho_{1}$ ( or $\eta_{1}$ ) and $b z_{2}^{\mathrm{R}}$ or $b p^{\mathrm{R}} / \mathrm{k} T$. From formula (9) for $\mu_{1} / \mathrm{k} T$, the definitions of $\eta_{1}, \eta_{2}$, and $q$ in (11) and (12), and the abbreviations $x$ and $y$ in (17), the exact result I for this one-dimensional model may be expressed in the form

$$
\frac{\mu_{1}}{\mathrm{k} T}-\ln \frac{\Lambda_{1}}{a}=\ln \frac{x / q}{1-y}+\frac{1}{q} \frac{x+y}{1-y} \quad(\mathrm{I}),
$$

equivalent to (19). Then also

$$
\frac{\mu_{1}^{0}}{\mathrm{k} T}-\ln \frac{\Lambda_{1}}{a}=\ln \frac{x}{q}+\frac{x}{q} .
$$

Then from (18), (22), and (24), the approximation IV is equivalently

$$
\frac{\mu_{1}}{\mathrm{k} T}-\ln \frac{\Lambda_{1}}{a}=\ln \frac{x}{q}+\frac{x}{q}+\frac{b p^{\mathrm{R}}}{\mathrm{k} T}\left(1+\frac{x+1}{q}\right) \mathrm{e}^{-x} \quad(\mathrm{IV}) .
$$

With $x$ as defined in (17), the right-hand side of (25) is explicitly a function only of $\eta_{1}$ for any given $q$ and $b p^{\mathrm{R}} / \mathrm{k} T$. The right-hand side of (23) may also be taken to be a function of $\eta_{1}$ for any given $q$ and $b p^{\mathrm{R}} / \mathrm{k} T$ provided that the $y$ that appears in it is understood to be that function of $x$ and $b p^{\mathrm{R}} / \mathrm{k} T$ given implicitly by (16), as remarked at the end of Section 3. In Fig. 2, the exact I and approximate IV are then plotted as functions of $\eta_{1}$ for $q=\frac{1}{4}$ and for $b p^{\mathrm{R}} / \mathrm{k} T=0.2,1.0$, and 4.0. To make a connection to Fig. 1, it may be noted that at $\eta_{1}=\frac{1}{4}$, for these three values of $b p^{\mathrm{R}} / \mathrm{k} T$ the volume fraction $\eta_{2}$ has the respective values $0.12,0.36$, and 0.59 .

At $b p^{\mathrm{R}} / \mathrm{k} T=0.2$ the exact and approximate functions of $\eta_{1}$ are indistinguishable on the scale of the figure and are shown as a single curve. At this $b p^{\mathrm{R}} / \mathrm{k} T$, as $\eta_{1}$ varies from 0.10 to 0.80 the volume fraction $\eta_{2}$ varies from 0.15 to 0.01 .

At $b p^{\mathrm{R}} / \mathrm{k} T=1.0$ the exact and approximate functions of $\eta_{1}$, while clearly distinguishable, are still very close over the range $0.10 \leqslant \eta_{1} \leqslant 0.80$. At this $b p^{\mathrm{R}} / \mathrm{k} T$, as $\eta_{1}$ varies from 0.10 to 0.80 the volume fraction $\eta_{2}$ varies from 0.44 to 0.06 .

At $b p^{\mathrm{R}} / \mathrm{k} T=4.0$ the exact and approximate values are very different (except near $\eta_{1}=0.67$, where the two curves happen to cross). The exact curve at this and all other values of $b p^{\mathrm{R}} / \mathrm{k} T$ has positive slope everywhere, as required by thermodynamic stability, and has the expected inflection where the curvature changes from negative to positive. The curve for the approximation at $b p^{\mathrm{R}} / \mathrm{k} T=4.0$, by contrast, just as 


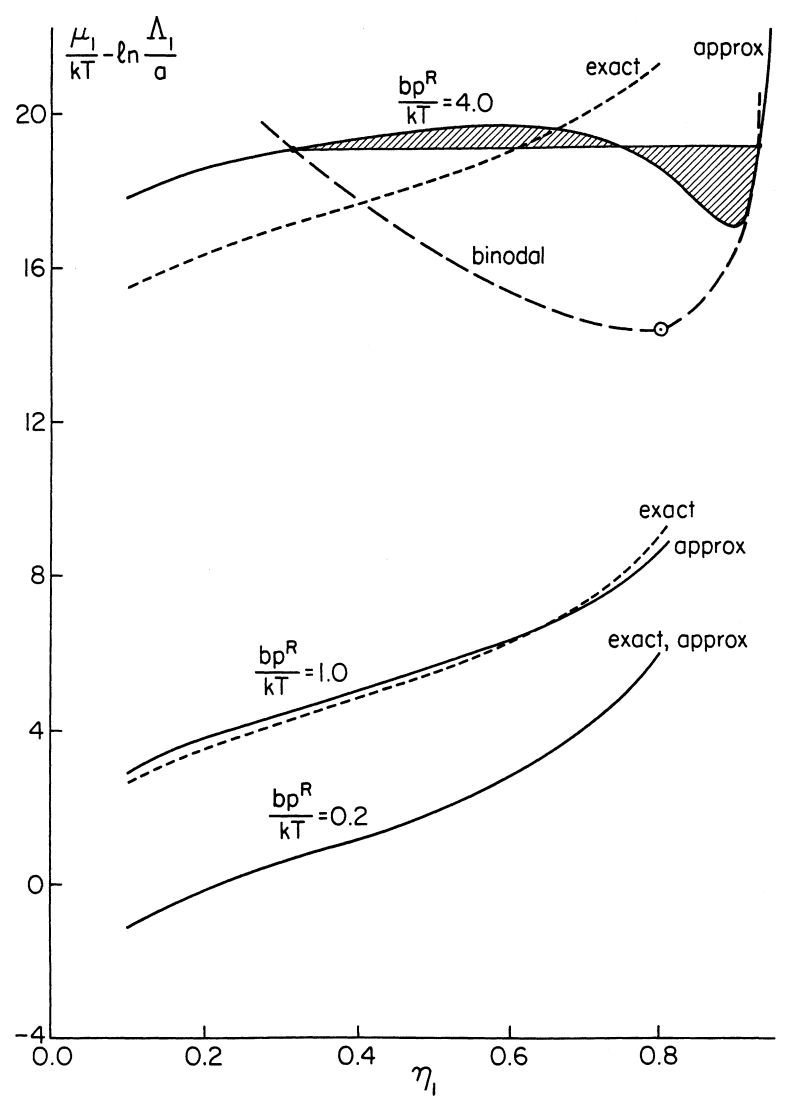

Fig. 2. The exact and approximate $\mu_{1} / \mathrm{k} T-\ln \left(\Lambda_{1} / a\right)$ as functions of the volume fraction $\eta_{1}$ for size ratio $q=b / a=\frac{1}{4}$ and for the fixed values $0.2,1.0$, and 4.0 of $b p^{\mathrm{R}} / \mathrm{k} T$. The curves "approx" are approximation IV. The binodal and critical point are those associated with the approximation.

it is about to show its inflection to positive curvature, instead develops a van der Waals loop. The equal-areas construction that gives the coexisting phases at this value of $b p^{\mathrm{R}} / \mathrm{k} T$, in this approximation, is also shown in the figure. This instability and the associated phase transition, occurring here in one dimension, are artifacts of the mean-field approximation that is implicit in those forms of the free-volume theories that are now being tested. The instability disappears in a more refined version of the free-volume theory in which some of the previously neglected correlations are taken into account [21]. Although the phase separation found here in this mean-field approximation is not truly realizable in one dimension, it is nevertheless of interest because it shows the propensity for phase separation in mixtures of hard particles of different sizes at high enough densities. The phase separation may then be realized in higher dimensions.

The binodal curve associated with the van der Waals loops is also shown in Fig. 2. The integration over $\eta_{1}$ of expression (25) for $\mu_{1} / \mathrm{k} T-\ln \left(\Lambda_{1} / a\right)$, which is required for 
the equal-areas construction, may be done analytically, with $x$ given in terms of $\eta_{1}$ by (17). Introduce the abbreviation

$$
\omega=b p^{\mathrm{R}} / \mathrm{k} T \text {. }
$$

Then if $\bar{\mu}_{1}$ is the common equilibrium value of $\mu_{1}$ in the two phases as given by the equal-areas rule, and if $x^{\prime}$ and $x^{\prime \prime}$ are the respective values of $x$ in the two coexisting phases, we have from (25), for any $\omega$ and $q$,

$$
\begin{aligned}
\frac{\bar{\mu}_{1}}{\mathrm{k} T}-\ln \frac{\Lambda_{1}}{a} & =\ln \frac{x^{\prime}}{q}+\frac{x^{\prime}}{q}+\omega\left(1+\frac{x^{\prime}+1}{q}\right) \mathrm{e}^{-x^{\prime}} \\
& =\ln \frac{x^{\prime \prime}}{q}+\frac{x^{\prime \prime}}{q}+\omega\left(1+\frac{x^{\prime \prime}+1}{q}\right) \mathrm{e}^{-x^{\prime \prime}},
\end{aligned}
$$

and from these and the equal-areas rule (after some simplification and rearrangement),

$$
\omega=\frac{\ln \left(x^{\prime} / x^{\prime \prime}\right)}{\mathrm{e}^{-x^{\prime \prime}}-\mathrm{e}^{-x^{\prime}}}=\frac{x^{\prime}-x^{\prime \prime}}{\left(1+x^{\prime \prime}\right) \mathrm{e}^{-x^{\prime \prime}}-\left(1+x^{\prime}\right) \mathrm{e}^{-x^{\prime}}} .
$$

It is the non-trivial root, $x^{\prime \prime} \neq x^{\prime}$, that is required in (27)-(29). These equations determine the binodal for any $q$. The binodal in the $\mu_{1} / \mathrm{k} T-\ln \left(\Lambda_{1} / a\right), \eta_{1}$ plane shown in Fig. 2 was calculated from (27)-(29) with $q=\frac{1}{4}$. At the critical point, $\mu_{1} / \mathrm{k} T-$ $\ln \left(\Lambda_{1} / a\right)=\ln (1 / q)+3 / q+1$ and $\eta_{1}=1 /(1+q)$, which for $q=\frac{1}{4}$, as in the figure, are 14.4 and 0.80 , respectively; while $\omega\left(=b p^{\mathrm{R}} / \mathrm{k} T\right)=\mathrm{e}=2.718 \ldots$ at the critical point, independently of $q$. Also at the critical point with $q=\frac{1}{4}$, the volume fraction $\eta_{2}=0.11$.

\section{Summary and conclusions}

The free-volume approximations (4)-(6) for the thermodynamics of mixtures of hard spheres of two different sizes have been tested by comparison with the exact results in one dimension. The comparisons are shown in Figs. 1 and 2.

One sees from Fig. 1 that approximation (6) (referred to in the text as IV), in which the interactions of the smaller spheres with each other is partially taken into account via the pressure $p^{\mathrm{R}}$ that such spheres would exert if they were alone in a reservoir at the exact activity $z_{2}^{\mathrm{R}}$ they have in the mixture, performs markedly better than either of the other two forms of the free-volume approximation (approximations II and III). For a size ratio $4: 1$ of larger to smaller, and a volume fraction $\eta_{1}=\frac{1}{4}$ of the larger spheres in the mixture, the deviation of the chemical potential $\mu_{1}$ of the larger spheres from its value $\mu_{1}^{0}$ when those spheres are alone in the volume, is closely approximated by IV for volume fractions $\eta_{2}$ of the smaller spheres up to about 0.70 , i.e., up to a total volume fraction $\eta_{1}+\eta_{2}$ that is about $95 \%$ of that of close packing.

The chemical potential $\mu_{1}$ as a function of the volume fraction $\eta_{1}$ at fixed $p^{\mathrm{R}}$, again for size ratio $4: 1$, is approximated very well by approximation IV for $b p^{\mathrm{R}} / \mathrm{k} T$ up to 1.0, as seen in Fig. 2. At this size ratio and at $\eta_{1}=\frac{1}{4}$ the value $b p^{\mathrm{R}} / \mathrm{k} T=1.0$ corresponds to $\eta_{2}=0.36$, i.e., to a total volume fraction $\eta_{1}+\eta_{2}=0.61$. At $b p^{\mathrm{R}} / \mathrm{k} T=\mathrm{e}=2.718 \ldots$ for 
any size ratio, this approximation IV leads to an instability (the critical point of phase separation). When the size ratio is $4: 1$ the volume fractions $\eta_{1}$ and $\eta_{2}$ at the critical point are 0.80 and 0.11 , respectively. This critical point produced by the approximation, while unphysical in one dimension, perhaps prefigures the (stable or metastable) phase separation of such mixtures in three dimensions.

\section{Acknowledgements}

BW is grateful to the Institute of Theoretical Physics and to the Debye Institute of the University of Utrecht for their hospitality in the fall of 1999. His work at Cornell was supported by the U.S. National Science Foundation and the Cornell Center for Materials Research. The authors gratefully acknowledge helpful conversations with Gerrit Vliegenthart, Martijn Oversteegen, Marjolein Dijkstra, René van Roij, and Robert Evans.

\section{References}

[1] S. Asakura, F. Oosawa, J. Chem. Phys. 22 (1954) 1255.

[2] A. Vrij, Pure Appl. Chem. 48 (1976) 471.

[3] A.P. Gast, C.K. Hall, W.B. Russell, J. Colloid Interface Sci. 96 (1983) 251.

[4] H.N.W. Lekkerkerker, W.C.K. Poon, P. Pusey, A. Stroobants, P. Warren, Europhys. Lett. 20 (1992) 559.

[5] F. Leal Calderon, J. Bibette, J. Bias, Europhys. Lett. 23 (1993) 653.

[6] S.M. Ilett, A. Orrock, W.C.K. Poon, P. Pusey, Phys. Rev. E 51 (1995) 1344.

[7] E.J. Meijer, D. Frenkel, J. Chem. Phys. 100 (1994) 6873.

[8] M. Dijkstra, J.M. Brader, R. Evans, J. Phys.: Condens. Matter 11 (1999) 10079.

[9] H.N.W. Lekkerkerker, A. Stroobants, Physica A 195 (1993) 387.

[10] W.C.K. Poon, P.B. Warren, Europhys. Lett. 28 (1994) 513.

[11] T. Biben, J.P. Hansen, Phys. Rev. Lett. 66 (1991) 2215.

[12] J.S. van Duijneveldt, A.W. Heinen, H.N.W. Lekkerkerker, Europhys. Lett. 21 (1993) 369.

[13] P.D. Kaplan, J.L. Rouke, A.G. Yodh, D.J. Pine, Phys. Rev. Lett. 72 (1994) 582.

[14] A. Imhof, J.K.G. Dhont, Phys. Rev. Lett. 75 (1995) 1662.

[15] M. Dijkstra, R. van Roij, R. Evans, Phys. Rev. Lett. 82 (1999) 117.

[16] T. Biben, P. Bladon, D. Frenkel, J. Phys.: Condens. Matter 8 (1996) 10799.

[17] M. Dijkstra, R. van Roij, R. Evans, Phys. Rev. Lett. 81 (1999) 2268.

[18] M. Dijkstra, R. van Roij, R. Evans, Phys. Rev. E 59 (1999) 5744.

[19] J.L. Lebowitz, E. Helfand, E. Praestgaard, J. Chem. Phys. 43 (1965) 774.

[20] A. Heck, Introduction to Maple, Springer, Berlin, 1993, p. 157.

[21] H.N.W. Lekkerkerker, S.M. Oversteegen, in preparation. 